\title{
Comments on "The 'Information' Concept in FRISCO Compared to Empirical Studies of Information in Decision Making" by J. Kaasbøll
}

\author{
Deborah Bunker \\ Deborah Bunker, School of Information Systems, Technology and Management, \\ University of New South Wales, Kensington, NSW 2052, Australia. \\ d.bunker@unsw.edu.au
}

The author's discussion of FRISCO, and its approach to "building a conceptual platform for the subject of information systems" raises many interesting issues for our field of research and practice. The need for "precise definitions" in our field, is highlighted due to the nature of the technology which supports information systems (IS). This point raises the issue of the many different perspectives, users of a single computer based IS (CBIS) might have, and how these can be accommodated in its design and creation. The idea of "rational ideals and descriptions of information use" is supported by the characteristics of an IS and the supporting technology, but are the ideals and descriptions of CBIS use purely rational?

There are many participants within an organisational framework who are not only required to make decisions but also act out cultural, political and social agendas. The author feels that "clarifying assumptions behind central concepts" is the way forward for more successful system design, and this idea is also a key motivation for FRISCO, however, cultural, political and social differences (all of which affect what each individual means by information) also need to be addressed as part of an overall systems building approach [Baskerville [1995]].

A way forward for the author's ideas and the FRISCO approach might be to look at the way we use language as a symbolic representation of culture [Young [1971]]. The author states that "a sender [of information] intends the 
receivers to act in some way on the basis of the information received, such that the sender should refer to the same semantic contents as the receiver". Language and the values, beliefs and norms on which it is based, therefore, shape information and the system that delivers it. The tools we use both physically/conceptually to create and use CBIS are also passed from one generation to the next through the use of the language [Young [1971], Bunker[1998]]. FRISCO can be seen, therefore, as a conceptual tool based on certain cultural underpinnings (use of language) which need to be understood for diffusion purposes.

The author also looks at the role of information, data, the relationship between them and how expertise, learning and observation affect perception, action, knowledge and interpretation. The cultural aspects of expertise (experts versus generalists), learning (polychronic/cyclical versus monochronic/linear) and observation (revealed truth versus experimentation) are all areas that the IS discipline needs to address with some rigor in order to ensure that tools, such as FRISCO, are well understood and applied in the most effective way [Hofstede [1998], Schein [1984]].

The author states that "Knowledge of intentions, meanings, values, rules, etc, has to be inferred from behavioural patterns." Do CBIS tools force us into a certain method of operation which infers certain behavioural patterns? If we consider, that even though we know that it is possible to create Web sites that are non-English language based, the origin, history and nature of the Internet and its use as a means of globalisation, necessitates the use of the originator's own cultural symbolic communication (language) as the communications "protocol" [Pargman [1998]].

In reading this paper there appears to be two specific areas that may be appropriate topics for further research. What are the values, norms and beliefs which underpin FRISCO? Understanding the motivation and assumptions on which FRISCO is based, will ensure that it is used appropriately. What are the values, norms and beliefs which underpin CBIS? It is only in understanding what these tools are attempting to represent, that we can create and use them in an effective manner [Bunker [1998]]. Too many systems have been created, promising success, which have then gone onto fail while consuming a vast amount of organisational resources.

In conclusion the author discusses the FRISCO approach and how attempts at applying it highlight the richness of social phenomona, thus limiting its applicability. This is by no means a negative statement. FRISCO's strength lies within the detailing of the values, norms and beliefs on which it is based. The articluation and understanding of these assumptions should ensure that it is used appropriately. Let us consider part 
of FRISCO's motivation. Why is there the need for such a framework? We have seen that historically we have had little success in the creation and design of systems and also there have been issues relating to the legitimisation of the IS "discipline". FRISCO provides us with an example of a well constructed and documented tool to utilise and study within our disciplinary area, thus giving us an opportunity to gain insight and knowledge.

\section{References}

Baskerville, R. [1995] "Structural entropy model of technology transfer" in Kautz, K., PriesHeje, J., Larsen, T. \& Sorgaard, P. (Eds), Proceedings of the First IFIP TC8 Working Conference on Diffusion and Adoption of Information Technology, October, 14-17,1995, Leangkollen, Oslo, Norway. Conference Notebook.

Bunker, D.J. [1998] "A Philosophy of Information Technology and Systems (IT \& S) as Tools:Tool Development Context, Associated Skills and the Global Technical Transfer (GTT) Process." MIS - shaped or misshaped ? Emerging issues in the new global arena. Proceedings of IFIP WG 8.7 Working Conference, Helsinki, Finland, 1998.

Hofstede, G. [1998] 'Attitudes, Values and Organisational Culture: Disentangling the Concepts' Organisation Studies, 19/3.

Pargman, D. [1998] "Reflections on Cultural Bias and Adaptation" from C. Ess and F. Sudweeks (eds), Proceedings of Cultural Attitudes Towards Communication and Technology '98, University of Sydney, Australia.

Schein, Edgar H. [1984] Coming to a New Awareness of Organisational Culture, Sloan Management Review, Winter.

Young [1971] An Introduction to the Study of Man, Oxford, Clarendon Press. 